\title{
Stability of Hybrid Automata with Average Dwell Time: An Invariant Approach
}

\author{
Sayan Mitra and Daniel Liberzon
}

\begin{abstract}
A formal method based technique is presented for proving the average dwell time property of a hybrid system, which is useful for establishing stability under slow switching. The Hybrid Input/Output Automaton (HIOA) of [12] is used as the model for hybrid systems, and it is shown that some known stability theorems from system theory can be adapted to be applied in this framework. The average dwell time property of a given automaton is formalized as an invariant of a corresponding transformed automaton, such that the former has average dwell time if and only if the latter satisfies the invariant. Formal verification techniques can be used to check this invariance property. In particular, the HIOA framework facilitates inductive invariant proofs by systematically breaking them down into cases for the discrete actions and continuous trajectories of the automaton. The invariant approach to proving the average dwell time property is illustrated by analyzing the hysteresis switching logic unit of a supervisory control system.
\end{abstract}

Index Terms-Average dwell time, Hybrid systems, Hybrid I/O automaton, Hysteresis Switching, Invariant, Stability.

\section{INTRODUCTION}

Systems with both discrete and continuous dynamics are called hybrid systems. Computer scientists have concentrated on verification of hybrid systems, and have developed a wide range of techniques for proving safety properties, from model checking (see, e.g., [1] and [7]) which is automatic but limited to moderate sized linear hybrid systems, to interactive theorem proving [2], [6], which is applicable to larger and more complicated hybrid systems. Control theorists, on the other hand, have viewed hybrid systems as switched systems or as dynamical systems with special boolean variables, and have addressed stability, controllability, and controller synthesis of such systems [18], [10]. The differences in these approaches espoused different terminologies and mathematical models, which has led to a lack of interaction between the two communities and isolated developments.

A platform bridging the gap by allowing computer scientists and control theorists to apply their techniques in the same modeling framework is desirable. To this end, we introduce the Hybrid Input/Output Automaton (HIOA) of [12] to the Control Systems community. HIOA is a mathematical model for developing compositional specifications

This work is supported by the MURI project:DARPA/AFOSR MURI F49620-02-1-0325 grant.

S. Mitra is with the Computer Science and Artificial Intelligence Laboratory, of Massachusetts Inst. of Technology, 32 Vassar Street, Cambridge, MA 02139, USA. Email: mitras@csail.mit.edu

D. Liberzon is with the Coordinated Science Laboratory, Univ. of Illinois at Urbana-Champaign, Urbana, IL 61821, U.S.A. Email: liberzon@uiuc. edu for a very general class of hybrid systems and it subsumes the class of untimed and timed distributed systems. Hybrid behavior is modeled as an alternating sequence of actions and trajectories; the actions correspond to discrete state transitions and the trajectories capture continuous evolution of the state variables of an automaton. Owing to this structure, safety properties which are also invariants of HIOA, can be proved inductively by a systematic case analysis of the automaton's actions and trajectories. Most of the prior work with HIOA focused on verifying safety of hybrid systems (see, e.g., [16], [11]).

In this paper we demonstrate how formal methods and the HIOA framework can be useful for proving invariants arising in stability analysis of hybrid systems. First, we show the straightforward adaptation of some known stability theorems from system theory to the HIOA framework. Then, we show that the task of proving the average dwell time property [9] which is used to prove stability of hybrid systems under slow switching, can be reduced to checking a set of invariants. We have chosen the average dwell time property to demonstrate the invariant approach because it decouples the problem of finding the Lyapunov functions (which we assume are given), from the problem of checking that all the executions of the HIOA satisfy certain properties. In general, properties of the executions of an automaton are harder to prove than invariant properties which are properties of the state. We transform the given HIOA $\mathcal{A}$ to a new HIOA $\mathcal{A}^{\prime}$ and find a condition $\mathcal{I}$ on the states of $\mathcal{A}^{\prime}$, such that $\mathcal{A}$ satisfies the average dwell time property if and only if $\mathcal{I}$ is an invariant of $\mathcal{A}^{\prime}$. This enables us to prove the average dwell time property by checking $\mathcal{I}$ with a suitable formal verification technique. We illustrate our approach by analyzing the stability of the hysteresis switching logic unit in a supervisory control system. In this case study we have proved the invariants by hand; however, our long term goal is to develop an integrated system which uses automatic theorem provers to efficiently verify the invariants arising in stability analysis of hybrid systems.

The rest of this paper is organized as follows: In Section II we describe the HIOA model, in Section III we define the various notions of stability and restate some known stability theorems in the HIOA framework. In Section IV we formalize the average dwell time property as a set of invariants. In Section V we present the analysis of the hysteresis switching unit of a supervisory control and we conclude in Section VI with a note on future research directions. Owing to space limitations, some of the theorems 
and invariants are stated without proof in this paper. Details can be found in the extended version of the paper [15].

\section{MAThematical PRELiminaries}

The hybrid I/O automaton framework of [12] evolved from the generalization of the timed $I / O$ automaton model [13] for real time distributed systems. A hybrid I/O automaton models hybrid behavior in terms of discrete transitions and continuous evolution of its state variables. Let $V$ be the set of variables of automaton $\mathcal{A}$. Each $v \in V$ is associated with a (static) type which is the set of values $v$ can assume. A valuation $\mathbf{v}$ for $V$ is a function that associates each variable $v \in V$ to a value in type $(v)$. The set of all valuations of $V$ is denoted by $\operatorname{val}(V)$. A restriction of $\mathbf{v}$ to a subset of variables $S \subset V$ will be denoted by v. $S$.

A trajectory $\tau$ of $V$ is a mapping $\tau: J \rightarrow \operatorname{val}(V)$, where $J$ is a left closed interval of time. The domain of $\tau$ is the interval $J$ and is denoted by $\tau$.dom. The first time of $\tau$ is the infimum of $\tau$.dom, also written as $\tau$.ftime. If $\tau$.dom is right closed then $\tau$ is closed and its limit time is the supremum of $\tau$.dom, also written as $\tau$.ltime.

Each variable $v \in V$ is also associated with a dynamic type (or dtype) which is the set of trajectories that $v$ may follow. Dynamic type dtype $(v)$ of a continuous (discrete) variable $v$ is the pasting closure of continuous (constant) functions from left closed intervals of time to type $(v)$.

\section{A. HIOA Model}

A hybrid $\mathrm{I} / \mathrm{O}$ automaton $\mathcal{A}$ consists of :

1) A set $V$ of variables, partitioned into internal $X$, input $U$, and output variables $Y$. The internal variables are also called state variables. The set $W=U \cup Y$ is the set of external variables. And, the set $Z \triangleq X \cup Y$ is called the set of locally controlled or local variables.

2) A set $A$ of actions, partitioned into internal $H$, input $I$, and output actions $O$.

3) A set of states $Q \subseteq \operatorname{val}(X)$,

4) A non-empty set of start states $\Theta \subseteq Q$,

5) A set of discrete transitions $\mathcal{D} \subseteq Q \times A \times Q$. A transition $\left(\mathbf{x}, a, \mathbf{x}^{\prime}\right) \in \mathcal{D}$ is written in short as $\mathbf{x} \stackrel{a}{\rightarrow} \mathcal{A} \quad \mathbf{x}^{\prime}$. The subscript is sometimes omitted and written as $\mathbf{x} \stackrel{a}{\rightarrow} \mathbf{x}^{\prime}$ when the automaton $\mathcal{A}$ is clear from the context.

6) A set of trajectories $\mathcal{T}$ for $V$, such that for every trajectory $\tau$ in $\mathcal{T}$, and for every $t \in \tau$.dom, $\tau(t) . X \in Q$ and $\mathcal{T}$ is closed under prefix, suffix, and concatenation. The first state $\tau(0) . X$ of trajectory is denoted by $\tau$.fstate. If $\tau$.dom is finite then $\tau$.lstate $=\tau(\tau$.ltime $) . X$.

Further, $\mathcal{A}$ is: (1) input action enabled, that is, it cannot block input actions, and (2) input trajectory enabled, that is, it accepts any trajectory of the input variables either by allowing time to progress for the entire length of the trajectory or by reacting with some internal action before that. As HIOA imposes few natural restrictions on its trajectories, it is capable of modeling a large class of hybrid systems. In particular it subsumes the class of hybrid automata used in [1].

For this paper we add the following extra assumptions to the HIOA model of [12]: (1) all variables are either discrete or continuous. For a set of variables $S$, we denote its discrete and continuous subsets by $S_{d}$ and $S_{c}$, and the corresponding state vectors by $\mathbf{s}_{d}$ and $\mathbf{s}_{c}$. And, (2) discrete transitions do not change the valuation of the continuous variables, that is, if $\mathbf{x} \stackrel{a}{\rightarrow} \mathbf{x}^{\prime}$, then $\mathbf{x} \cdot \mathbf{x}_{c}=\mathbf{x}^{\prime} \cdot \mathbf{x}_{c}$. These assumptions are made for simplicity and bring our model closer to the model of switched systems considered in [10].

\section{B. Executions and Invariants}

An execution fragment of $\mathcal{A}$ is a (possibly infinite) sequence of actions and trajectories $\alpha=\tau_{0}, a_{1}, \tau_{1}, a_{2} \ldots$, where each $\tau_{i} \in \mathcal{T}, a_{i} \in A$, and if $\tau_{i}$ is not the last trajectory in $\alpha$ then $\tau_{i}$ is finite and $\tau_{i}$.lstate $\stackrel{a_{i+1}}{\longrightarrow} \tau_{i+1}$.f state. For an execution fragment $\alpha$, the first state $\alpha . f$ state $=\tau_{0} . f$ state, likewise $\alpha$.ftime $=\tau_{0}$.ftime. An execution fragment is closed if it is a finite sequence and the domain of the final trajectory is a finite closed interval. The length of a closed execution fragment is the number of elements (actions and trajectories) in the sequence and its limit time $\alpha$.ltime is $\tau_{n}$.ltime, where $\tau_{n}$ is the last trajectory of $\alpha$. The duration of a closed execution fragment is its length in time and is defined as $\alpha . d u r=\sum_{i=0}^{n}\left(\tau_{i}\right.$.ltime $-\tau_{i}$. ftime $)$. We denote the valuation of the continuous variables $X_{c}$ at time $t, \alpha$.ftime $\leq t \leq \alpha$.ltime, in the execution fragment $\alpha$ by $\alpha(t)$. Note that $\alpha(t)$ is uniquely determined because the discrete actions do not alter the valuation of the continuous variables. An execution fragment $\alpha$ is an execution if $\alpha$.f state $\in \Theta$. A state of $\mathcal{A}$ is reachable if it is the last state of some closed execution. An execution fragment $\alpha$ is reachable if $\alpha$.f state is reachable.

An invariant property of $\mathcal{A}$ is a condition on $V$ that remains true in all reachable states of $\mathcal{A}$. The structure of HIOA allows systematic proof of invariants. An invariant $\mathcal{I}$ is either derived from other invariants or proved by induction on the length of a closed execution of $\mathcal{A}$ as follows:

1) base step: $\mathcal{I}(s)$ is true for all $s \in \Theta$,

2) induction step: (a) discrete part: for every discrete transition $s \stackrel{\pi}{\rightarrow} s^{\prime}, \mathcal{I}(s)$ implies $\mathcal{I}\left(s^{\prime}\right)$, and (b) continuous part: for any closed trajectory $\tau \in \mathcal{T}$, with $\tau$. f state $=s$ and $\tau$.lstate $=s^{\prime}, \mathcal{I}(s)$ implies $\mathcal{I}\left(s^{\prime}\right)$.

This structure is particularly helpful in organizing large, complex proofs and for automating invariant proofs in a theorem prover.

\section{STABILITy TheOREMS IN HIOA FrAmEWORK}

In this section we define what it means for a HIOA $\mathcal{A}$ to be stable. Here and in the following section, we are concerned with hybrid systems with no continuous inputs, and we assume that there exists a family of sufficiently regular (locally Lipschitz) functions $f_{p}: \mathrm{R}^{n} \rightarrow \mathrm{R}^{n}, p \in \mathcal{P}$, 
such that every trajectory of $\mathcal{A}$ satisfies $\dot{\mathbf{x}}_{c}=f_{p}\left(\mathbf{x}_{c}\right)$ for some $p \in \mathcal{P}$, where $\mathcal{P}$ is a finite index set.

\section{A. Stability Definitions}

Let us assume that all the subsystems of $\mathcal{A}$ have the origin as their common equilibrium point, that is, $f_{p}(0)=0$ for all $p \in \mathcal{P}$. The origin is a stable equilibrium point of a HIOA $\mathcal{A}$, in the sense of Lyapunov, if for every $\epsilon>0$, there exists a $\delta>0$, such that for every execution $\alpha$ of $\mathcal{A}$, we have

$$
|\alpha(0)| \leq \delta \Rightarrow|\alpha(t)| \leq \epsilon \quad \forall t \quad 0 \leq t \leq \alpha . \text { ltime },
$$

and we say that $\mathcal{A}$ is stable. A HIOA $\mathcal{A}$ is asymptotically stable if it is stable and $\delta$ can be chosen so that

$$
|\alpha(0)| \leq \delta \Rightarrow \alpha(t) \rightarrow 0 \quad \text { as } t \rightarrow \infty
$$

If the above condition holds for all $\delta$ then $\mathcal{A}$ is globally asymptotically stable.

Uniform stability is a concept which guarantees that the stability property in question holds, not just for executions, but for any execution fragment. Therefore, $\mathcal{A}$ is uniformly stable in the sense of Lyapunov, if for every $\epsilon>0$ there exists a constant $\delta>0$, such that for any execution fragment $\alpha$,

$\left|\alpha\left(t_{0}\right)\right| \leq \delta \Rightarrow|\alpha(t)| \leq \epsilon, \forall t_{0}, t, 0 \leq t_{0} \leq t \leq \alpha$. ltime

A HIOA $\mathcal{A}$ is said to be uniformly asymptotically stable if it is uniformly stable and there exists a $\delta>0$, such that for every $\epsilon>0$ there exists a $T$, such that for any execution fragment $\alpha$,

$$
\left|\alpha\left(t_{0}\right)\right| \leq \delta \Rightarrow|\alpha(t)| \leq \epsilon, \quad \forall t \geq t_{0}+T
$$

It is said to be globally uniformly asymptotically stable if the above holds for all $\delta$, with $T=T(\delta, \epsilon)$.

All the above stability properties are by definition uniform over executions. We will also make use of the following weaker notion of stability: a given execution is stable (uniformly stable, asymptotically stable, etc.) if the corresponding property is satisfied for this execution.

\section{B. Common Lyapunov Function}

The basic tool for studying uniform stability of hybrid systems relies on the existence of a single Lyapunov function whose derivative along the trajectories of all the subsystems in $\mathcal{P}$ satisfies the suitable inequalities.

Definition 1. Given a positive definite continuously differentiable function $V: \mathrm{R}^{n} \rightarrow \mathrm{R}^{n}$, we say that it is a common Lyapunov function for a HIOA $\mathcal{A}$ if there exists a positive definite continuous function $W: \mathrm{R}^{n} \rightarrow \mathrm{R}^{n}$, such that we have

$$
\frac{\partial V}{\partial \mathbf{x}_{c}} f_{p}\left(\mathbf{x}_{c}\right) \leq-W\left(\mathbf{x}_{c}\right) \quad \forall \mathbf{x}_{c}, \quad \forall p \in \mathcal{P}
$$

Theorem 1. If a HIOA $\mathcal{A}$ has a radially unbounded common Lyapunov function then $\mathcal{A}$ is globally uniformly asymptotically stable.

\section{Multiple Lyapunov Functions}

In the absence of a common Lyapunov function for all the subsystems in $\mathcal{P}$, the stability of HIOA in general depends on the choice of an execution. Multiple Lyapunov functions [3] is an useful tool for proving stability of a chosen execution. In this case, each subsystem $p \in \mathcal{P}$ is associated with a Lyapunov function $V_{p}$, and one attempts to prove the stability of the execution using the continuous decay of the $V_{p}$ 's and the switching logic between the subsystems. In control theory literature [10], [9] the switches between the subsystems $p \in \mathcal{P}$ are defined in terms of a "switching signal" which is a piece-wise constant function $\sigma:[0, \infty) \rightarrow \mathcal{P}$. In the HIOA model the switches are defined by the discrete transitions of the automaton, so we define the notion of switching times as follows:

Let $M: \mathcal{T} \rightarrow \mathcal{P}$ be a function that gives the index $p$ of the function $f_{p}$, which is active over the trajectory $\tau$. Whenever a discrete action $a_{i}$ occurs such that $M\left(\tau_{i-1}\right) \neq$ $M\left(\tau_{i}\right)$, the HIOA $\mathcal{A}$ is said to undergo a switch.

Definition 2. For any execution fragment $\alpha=\tau_{0} a_{1} \tau_{1} \ldots$, an instant of time $t \in \alpha$.dom is called a switching time if there exists $i$ such that $t=\tau_{i}$.ltime, and $M\left(\tau_{i}\right) \neq$ $M\left(\tau_{i+1}\right)$.

Theorem 2. Let $V_{p}$ be a radially unbounded Lyapunov function corresponding to the globally asymptotically stable system $\dot{x}=f_{p}(x)$ for each $p \in \mathcal{P}$. An execution $\alpha$ of a HIOA $\mathcal{A}$ is globally asymptotically stable if there exists a family of positive definite continuous functions $W_{p}, p \in \mathcal{P}$ such that, for every pair of switching times $t, t^{\prime}$ in $\alpha$, and the corresponding trajectories $\tau_{i}, \tau_{j}$, if $M\left(\tau_{i}\right)=M\left(\tau_{j}\right)=p$ and $M\left(\tau_{k}\right) \neq p, \forall k, i<k<j$ then $V_{p}\left(\tau_{j}\left(t^{\prime}\right)\right)-V_{p}\left(\tau_{i}(t)\right) \leq-W_{p}\left(\tau_{i}(t)\right)$.

\section{Stability Under Slow Switching}

It is well known that a switched system is stable if all the individual subsystems are stable and the switching is sufficiently slow, so as to allow the dissipation of the transient effects after each switch. The dwell time [17] and the average dwell time [9] criteria define restricted classes of switching signals, based on switching speeds, and one can conclude the stability of a system with respect to these restricted classes.

Definition 3. Let $t_{1}, t_{2}, \ldots$ be the switching times of an execution fragment $\alpha$ of a HIOA $\mathcal{A}$. The execution fragment $\alpha$ has a dwell time $\tau_{d}>0$ if it satisfies the inequality $t_{i+1}-t_{i} \geq \tau_{d}$, for all $i$. If all reachable execution fragments of $\mathcal{A}$ have dwell times $\geq \tau_{d}$ then $\mathcal{A}$ has a dwell time $\tau_{d}$.

Definition 4. Let $N(\alpha)$ denote the number of switches over an execution fragment $\alpha$ of a HIOA $\mathcal{A}$. The execution fragment has an average dwell time $\tau_{a}>0$ if there exists a positive number $N_{0}$ such that:

$$
N(\alpha) \leq N_{0}+\frac{\alpha \cdot d u r}{\tau_{a}} .
$$


If all reachable execution fragments of $\mathcal{A}$ have average dwell times $\geq \tau_{a}$ with a fixed $N_{0}$ then $\mathcal{A}$ has an average dwell time $\tau_{a}$.

The following theorem, adapted to the HIOA framework from the results in [9], uses the concept of average dwell time to give a sufficient condition for stability. Since dwell time is a special case of average dwell time with $N_{0}=1$, a separate theorem for dwell time is not necessary.

Theorem 3. Consider a HIOA $\mathcal{A}$ with its trajectories specified by a family of functions $f_{p}, p \in \mathcal{P}$. Suppose there exist positive definite, radially unbounded, and continuously differentiable functions $V_{p}: \mathrm{R}^{n} \rightarrow \mathrm{R}^{n}$, for each $p \in \mathcal{P}$, and positive numbers $\lambda_{0}$ and $\mu$ such that:

$$
\begin{aligned}
\frac{\partial V_{p}}{\partial \mathbf{x}_{c}} f_{p}\left(\mathbf{x}_{c}\right) & \leq-\lambda_{0} V_{p}\left(\mathbf{x}_{c}\right), \quad \forall \mathbf{x}_{c}, \quad \forall p \in \mathcal{P} \\
V_{p}\left(\mathbf{x}_{c}\right) & \leq \mu V_{q}\left(\mathbf{x}_{c}\right), \quad \forall \mathbf{x}_{c}, \quad \forall p, q \in \mathcal{P} .
\end{aligned}
$$

Then $\mathcal{A}$ is globally uniformly asymptotically stable if it has an average dwell time $\tau_{a}>\frac{\log \mu}{2 \lambda_{0}}$.

Theorem 3 roughly states that a hybrid system is uniformly stable if the discrete switches are between modes which are individually stable, provided that the switches do not occur too frequently on the average. This stability condition effectively allows us to decouple the construction of Lyapunov functions-one for each $p \in \mathcal{P}$, which we assume are known from available methods of system theory-from the problem of checking that every execution of the automaton satisfies Equation (5).

\section{Average Dwell Time: Invariant Approach}

In general, it is harder to prove properties of executions of automata than it is to prove invariants, which are properties of state. Several formal verification techniques have been developed expressly for checking invariants of hybrid automata (see [1], [7], [6], and Chapters 5 and 6 of [18]). So, once we have translated the average dwell time property to a set of invariant properties, we can appeal to the suitable formal verification tool for checking the invariants.

\section{A. Transformation for Stability Verification}

We transform the given HIOA $\mathcal{A}$ to a new HIOA $\mathcal{A}^{\prime}$ as follows: In addition to all the variables of $\mathcal{A}$, automaton $\mathcal{A}^{\prime}$ has two new internal variables: a counter $Q$ and a timer $t$, both initialized 0 . The counter $Q$ counts the number of mode switches, and the timer reduces the count by 1 in every $\tau_{a}$ time. For every discrete transition $s \stackrel{a}{\rightarrow} \mathcal{A} s^{\prime}$ of $\mathcal{A}$, automaton $\mathcal{A}^{\prime}$ has a corresponding transition $s \stackrel{a}{\rightarrow} \mathcal{A}^{\prime} s^{\prime}$, such that $s^{\prime} . Q=s . Q+1$. In addition $\mathcal{A}^{\prime}$ has internal action which occurs every $\tau_{a}$ time and decrements $Q$ by one. Finally, for every trajectory $\tau^{\prime}$ of $\mathcal{A}^{\prime}$, the restriction of $\tau$ on the set of continuous variables of $\mathcal{A}$ is a trajectory of $\mathcal{A}$, i.e., $\tau^{\prime} \downarrow Z_{c} \in \mathcal{T}_{\mathcal{A}}$, and $\dot{t}=1$.

Lemma 1. All closed executions of $\mathcal{A}$ satisfy Equation (5) if and only if $Q \leq N_{0}$ in all reachable states of $\mathcal{A}^{\prime}$.
Proof. Since $\alpha$ is a closed execution of $\mathcal{A}$, we can replace a.dur in Equation (5) with o.ltime. For the "if" part, consider a closed execution $\alpha$ of $\mathcal{A}$ and let $\alpha^{\prime}$ be the "corresponding" execution of $\mathcal{A}^{\prime}$. Let $s^{\prime}$ be the last state of $\alpha$, therefore from the invariant we know that $s^{\prime} . Q \leq N_{0}$. From construction of $\mathcal{A}^{\prime}$ we know that, $N(\alpha)=N\left(\alpha^{\prime}\right)$ and $\alpha^{\prime}$.ltime $=\alpha$.ltime and therefore $s^{\prime} . Q=N\left(\alpha^{\prime}\right)-$ $\left\lfloor\frac{\alpha^{\prime} \text {.ltime }}{\tau_{a}}\right\rfloor$. It follows that $N(\alpha)-\frac{\alpha . \text { ltime }}{\tau_{a}} \leq N_{0}$.

For the "only if" part, consider a reachable state $s^{\prime}$ of $\mathcal{A}^{\prime}$. There exists an execution $\alpha^{\prime}$ such that $s^{\prime}$ is the last state of $\alpha^{\prime}$. Let $\alpha$ be an execution of $\mathcal{A}$ "corresponding" to $\alpha^{\prime}$. Since $N(\alpha) \leq N_{0}+\left\lfloor\frac{\alpha . t i m e}{\tau_{a}}\right\rfloor$ implies $N\left(\alpha^{\prime}\right) \leq N_{0}+\left\lfloor\frac{\alpha^{\prime} \text {.ltime }}{\tau_{a}}\right\rfloor$, it follows that $s^{\prime} \cdot Q^{\tau_{a}} \leq N_{0}$.

Theorem 4. All executions of $\mathcal{A}$ satisfy Equation (5) if and only if $Q \leq N_{0}$ in all reachable states of $\mathcal{A}^{\prime}$.

Proof. We only have to show that if any execution $\alpha$ of $\mathcal{A}$ violates (5), then there exists a closed execution $\alpha^{\prime}$ of $\mathcal{A}$ that violates (5) as well. If $\alpha$ is infinite, then there is a closed prefix of $\alpha$ that violates (5). If $\alpha$ is finite and open, then the closed prefix of $\alpha$ excluding the last trajectory of $\alpha$ violates (5).

In (5), the number $N_{0}$ can be arbitrary. Thus to show that a given $\tau_{a}$ is an average dwell time of an execution, we need to show that $Q$ is bounded, while to show that it is an average dwell time of an automaton, we need to show that $Q$ is bounded uniformly over all executions.

\section{B. Transformation for Uniform Stability Verification}

The above transformation is acceptable for asymptotic stability, but it allows $Q$ to become negative, and then rapidly return to zero, so it does not guarantee uniform stability. For uniform stability we want all reachable execution fragments of $\mathcal{A}$ to satisfy (5).

Consider any reachable execution fragment $\alpha$ of $\mathcal{A}$, with $\alpha$.ftime $=t_{1}$, and $\alpha$.ltime $=t_{2}$. Let $N\left(t_{2}, t_{1}\right)$ and $Q\left(t_{2}, t_{1}\right)$ denote the number of switches and the number of "extra" switches over $\alpha$ with respect to dwell time $\tau_{a}$, that is, $Q\left(t_{2}, t_{1}\right)=N\left(t_{2}, t_{1}\right)-\left(t_{2}-t_{1}\right) / \tau_{a}$. Thus, every reachable execution fragment $\alpha$ of $\mathcal{A}$ satisfies (5), if

$$
\begin{gathered}
N\left(t, t_{0}\right)=Q(t, 0)+\frac{t}{\tau_{a}}-Q\left(t_{0}, 0\right)-\frac{t_{0}}{\tau_{a}} \leq N_{0}+\frac{t-t_{0}}{\tau_{a}} \\
\text { or, } \\
Q\left(t, t_{0}\right) \leq N_{0},
\end{gathered}
$$

where $t_{0}=\alpha$.ftime, and $t=\alpha$.ltime. So, we introduce an additional variable $Q_{\min }$ which stores the magnitude of the smallest value ever attained by $Q$. Then, for uniform stability we need to show that the total change in $Q$ between any two reachable states is bounded by $N_{0}$.

Theorem 5. All reachable execution fragments of $\mathcal{A}$ satisfy Equation (5), if and only if $Q-Q_{\min } \leq N_{0}$ in all reachable states of $\mathcal{A}^{\prime}$. 


\section{Hysteresis SWITCHING}

In this section the invariant based technique is applied to a hysteresis switching logic unit which is a subsystem of an adaptive supervisory control system taken from [8] (also Chapter 6 of [10]). Our goal is to prove the average dwell time property of this switching logic, which guarantees stability of the overall supervisory control system (see the above references for details).

An adaptive supervisory controller consists of a family of candidate controllers $u_{p}, p \in \mathcal{P}$, which correspond to the parametric uncertainty range of the plant in a suitable way. Such a controller structure is particularly useful when the parametric uncertainty is so large that robust control design tools are not applicable. The controller operates in conjunction with a set of on-line estimators that provide monitoring signals $\mu_{p}, p \in \mathcal{P}$; intuitively, smallness of $\mu_{p}$ indicates high likelihood that $p$ is the actual parameter value. Based on these signals, the switching logic unit generates, at each instant of time $t$, the index $\sigma(t)$ of the controller to be applied to the plant.

In building the HIOA model, we take as inputs the monitoring signals $\mu_{p}$ and focus on the switching logic unit which implements scale independent hysteresis switching as follows: at an instant of time when controller $r$ is operating, that is, $\sigma=r$ for some $r \in \mathcal{P}$, if there exists a $p \in \mathcal{P}$ such that $\mu_{p}(1+h) \leq \mu_{r}$ for some fixed hysteresis constant $h$, then the switching logic sets $\sigma=p$ and applies output of controller $p$ to the plant. Below we describe and analyze the HIOA representing this switching logic unit, which we call HysteresisSwitch automaton.

We consider a finite set of continuous, monotonically nondecreasing monitoring signals $\mu_{p}, p \in \mathcal{P}$ satisfying:

$$
\begin{aligned}
\mu_{p}(0) & \geq C_{0} \\
\mu_{p^{*}}(t) & \leq C_{1}+C_{2} e^{2 \lambda t}, \text { for some } p^{*} \in \mathcal{P}
\end{aligned}
$$

where $C_{0}, C_{1}$ and $C_{2}$ are positive constants. Equation (8) sets a lower bound on the initial values of all the monitoring signals, and Equation (9) states that there exists some $p^{*} \in$ $\mathcal{P}$ for which the corresponding monitoring signal satisfies the exponential upper bound.

\section{A. HIOA Specification}

The hysteresis switch is specified as a HIOA (Figure 1) in the style described in [16]. The variables of the automaton are declared and initialized in the variables section; each variable's name is followed by its type and its initial value. The analog keyword preceding a variable name indicates that it is a continuous variable. The input variables $\mu_{p}$, $p \in \mathcal{P}$ model the monitoring signals that are inputs to the switch. The discrete switching signal $\sigma$ is an output variable because it is visible to the outside word; remaining variables are internal to the automaton. The variables $c$ and $d$ count the number of switches and the number of $\tau_{a}$ periods elapsed. Variable $\mu_{p}^{i}$ stores the values of $\mu_{p}$ at the instant when $\sigma$ became equal to $p$ for the $i^{\text {th }}$ time; initially $\mu_{p}^{0}=\mu_{p}$, for all $p \in \mathcal{P}, \mu_{p}^{1}=\mu_{p}$, for $p=\sigma$, and the rest of the $\mu_{p}^{i}$ s are set to a null value $\perp$. The variable $c_{p}$ counts the number of intervals in which $\sigma$ equaled $p$; and $t_{p}$ is a reset timer measuring the length of the last such interval.

The discrete transitions section defines the two actions of the automaton, namely dequeue and switch,$p \in \mathcal{P}$. An action is enabled or in other words, it can occur when the condition following the precondition keyword is true. The change in the state variables when the action does occur is described by the effect part of the transition definition.

The trajectories section defines the evolution of the continuous variables in terms of the differential and algebraic equations. The $d($.$) in the evolve section stands$ for derivative. The stopping condition, in this automaton, is the disjunction of the action preconditions, so it forces the actions to occur whenever they are enabled.

\section{B. Invariant Properties}

In this section we state a sequence of invariants which lead to the target average dwell time property of the Hysteresisswitch automaton. As a representative invariant proof in the HIOA framework we present the proof of Invariant 2. The proofs of the other invariants are omitted owing to space constraints and can be found in the longer online version of the paper [15]. The first three invariants lead to give a lower bound on the change in the history variables $\left(\mu_{p}^{i}\right.$, s) necessary to perform a certain number of switches. And we already have an upper bound on the rate of growth of the monitoring signals from Equations (8) and (9). Putting these two pieces together in Invariant 5, and using Theorem 4 we derive the average dwell time property.

Invariant 1. $Q \leq c-\frac{n o w}{\tau_{a}}+1$.

Invariant 2. $\forall q \in \mathcal{P}$,

(1) $\sigma=q \Rightarrow \forall p \in \mathcal{P}, \mu_{q} \leq(1+h) \mu_{p}$,

(2) $\sigma=q \wedge c_{q}>0 \wedge t_{q}=0 \Rightarrow \forall p \in \mathcal{P}, \mu_{q} \leq \mu_{p}$.

Proof. Part(1): Initial states satisfy. For the induction step we need to consider only discrete transitions $s \stackrel{a}{\rightarrow} s^{\prime}$, where $a=$ switch $_{q}$. Let $s . \sigma=r$, we know that $s^{\prime} . \sigma=q$. By inductive hypothesis $s . \mu_{r} \leq(1+h) s . \mu_{p}$, for all $p \in \mathcal{P}$. By precondition of switch $q,(1+h) s . \mu_{q} \leq s . \mu_{r}$. By continuity of $\mu_{p}$ 's $(1+h) s^{\prime} \cdot \mu_{q} \leq s^{\prime} \cdot \mu_{r} \leq(1+h) s^{\prime} . \mu_{p}$, for all $p \in \mathcal{P}$.

From the above it follows that $s^{\prime} \cdot \mu_{q} \leq(1+h) s^{\prime} \cdot \mu_{p}$, for all $p \in \mathcal{P}$. The stopping condition of activity flow ensures that the invariant is preserved over all trajectories.

Part(2) : Initial states satisfy the invariant because $q=$ $\arg \min _{p \in \mathcal{P}} \mu_{p}$. For the induction step, consider a discrete transition $s \stackrel{a}{\rightarrow} s^{\prime}$, where $a=$ switch $_{q}$. Let $s . \sigma=r$, we know that $s^{\prime} . \sigma=q$. From Part (1), s. $\mu_{r} \leq(1+h) s . \mu_{p}$, for all $p \in \mathcal{P}$. By precondition of switch ${ }_{q},(1+h) s . \mu_{q} \leq s . \mu_{r}$, and by continuity of $\mu_{p}$ 's, $s^{\prime} \cdot \mu_{q} \leq s^{\prime} . \mu_{p}$, for all $p \in \mathcal{P}$

We note that the dequeue actions do not alter any of the variables involved in the invariant. Now, consider any trajectory $\tau$. If $\tau$ is a point trajectory, then the invariant holds. If $\tau$ is not a point trajectory, then the invariant holds vacuously because $\tau$.lstate. $t_{q} \neq 0$. 

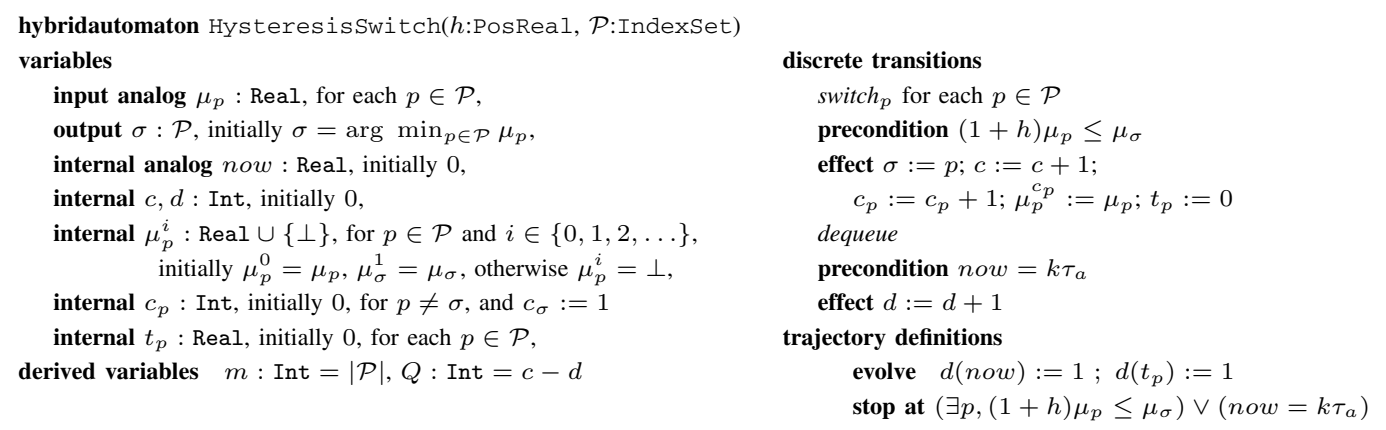

Fig. 1. HIOA specification of the hysteresis switching logic in the supervisory controller

Invariant 3. $\forall q \in \mathcal{P}, c_{q} \geq 2 \Rightarrow \mu_{q}^{c_{q}} \geq(1+h) \mu_{q}^{c_{q}-1}$.

Invariant 4. $\exists q \in \mathcal{P}$ such that $c_{q} \geq\left\lceil\frac{c-1}{m}\right\rceil$.

Invariant 5. If we set $\tau_{a}$ to $\frac{\log (1+h)}{2 \lambda m}$ then,

$$
Q \leq 2+m+\frac{m}{\log (1+h)} \log \left(\frac{C_{1}+C_{2}}{C_{0}}\right)
$$

Theorem 6. The HysteresisSwitch automaton has an average dwell time of at least $\frac{\log (1+h)}{2 \lambda m}$.

To ensure stability of the overall supervisory control system, the parameters $h$ and $\lambda$ must be such that this average dwell time satisfies the inequality of Theorem 3 .

\section{REMARKS AND FUTURE WORK}

We have introduced the hybrid I/O automaton framework as a modeling platform in which analysis techniques from both computer science and control theory can be applied. To demonstrate its utility and expressive power, we have shown how known stability theorems from system theory literature can be adapted and applied in this framework. Then, we formalized the average dwell time property of hybrid systems as a set of invariants, thereby making it possible to prove (uniform) stability of hybrid systems under slow switching using formal verification techniques. The suggested method has been illustrated by analyzing the stability of a hysteresis switching logic unit in a supervisory control system.

In this paper we examined internal stability only; however, the explicit external variables of HIOA make the framework suitable for studying input-output properties of hybrid systems as well. Secondly, the hand-proofs of invariants can be partially-mechanized with theorem provers, as shown in [2], [14]. Another direction of future research is to extend these techniques to stochastic hybrid systems, by combining the probabilistic IOA model of [5] with stability results for stochastic switched systems from [4].

Acknowledgements. We are grateful to Nancy Lynch for helpful discussions.

\section{REFERENCES}

[1] R. Alur, C. Courcoubetis, N. Halbwachs, T. A. Henzinger, P.-H. Ho, X. Nicollin, A. Olivero, J. Sifakis, and S. Yovine. The algorithmic analysis of hybrid systems. Theoretical Computer Science, 138(1):334, 1995.

[2] M. Archer, C. Heitmeyer, and S. Sims. TAME: A PVS interface to simplify proofs for automata models. In Proceedings of UITP '98, July 1998.

[3] M. Branicky. Multiple lyapunov functions and other analysis tools for switched and hybrid systems. IEEE Transactions on Automatic Control, 43:475-482, 1998.

[4] D. Chatterjee and D. Liberzon. On stability of stochastic switched systems. in Proceedings of the 43rd Conference on Decision and Control. Dec 2004, to appear.

[5] L. Cheung, N. A. Lynch, R. Segala, and F. Vaandrager. Switched probabilistic i/o automata. Submitted for publication, July 2, 2004.

[6] C. Heitmeyer and N. A. Lynch. The generalized railroad crossing: A case study in formal verification of real-time system. In Proceedings of the 15th IEEE Real-Time Systems Symposium, San Juan, Puerto Rico, December 1994. IEEE Computer Society Press.

[7] T. A. Henzinger. The theory of hybrid automata. In Proceedings of the 11th Annual IEEE Symposium on Logic in Computer Science (LICS '96), pages 278-292, New Brunswick, New Jersey, 1996.

[8] J. P. Hespanha, D. Liberzon, and A.S. Morse. Hysteresis-based switching algorithms for supervisory control of uncertain systems. Automatica, 39:263-272, 2003.

[9] J. P. Hespanha and A. Morse. Stability of switched systems with average dwell-time. In Proceedings of 38th IEEE Conference on Decision and Control, pages 2655-2660, 1999.

[10] D. Liberzon. Switching in Systems and Control. Systems and Control: Foundations and Applications. Birkhauser, Boston, June 2003.

[11] C. Livadas, J. Lygeros, and N. A. Lynch. High-level modeling and analysis of TCAS. In Proceedings of the 20th IEEE RealTime Systems Symposium (RTSS'99),Phoenix, Arizona, pages 115125, December 1999.

[12] N. A. Lynch, R. Segala, and F. Vaandrager. Hybrid I/O automata. Information and Computation, 185(1):105-157, August 2003.

[13] N. A. Lynch and F. Vaandrager. Forward and backward simulations - part II: Timing-based systems. Information and Computation, 128(1):1-25, July 1996.

[14] S. Mitra and M. Archer. Reusable PVS proof strategies for proving abstraction properties of I/O automata. In IJCAR Workshop on strategies in automated deduction, Cork, Ireland, July 2004.

[15] S. Mitra and D. Liberzon. Stability of hybrid automata with average dwell time: an invariant approach. http://theory.lcs.mit.edu/ mitras/research/cdc04-full.ps.gz.

[16] S. Mitra, Y. Wang, N. A. Lynch, and E. Feron. Safety verification of model helicopter controller using hybrid Input/Output automata. In HSCC'03, Hybrid System: Computation and Control, Prague, the Czech Republic, April 3-5 2003.

[17] A. S. Morse. Supervisory control of families of linear set-point controllers, part 1: exact matching. IEEE Transactions on Automatic Control, 41:1413-1431, 1996.

[18] A. van der Schaft and H. Schumacher. An Introduction to Hybrid Dynamical Systems. Springer, London, 2000. 